\title{
Guía para la definición de los perfiles competenciales profesionales
}

Maria Nolla-Domenjó, Arcadi Gual, Luis Pallarés, Jordi Palés-Argullós

La declaración de Bolonia de 1999 ha comportado diferentes cambios y retos en el mundo de la educación médica. Entre ellos, uno de los más relevantes es la elaboración de programas formativos basados en competencias evaluadas, también denominados programas formativos basados en resultados.

Los programas formativos basados en competencias evaluadas o resultados se caracterizan por describir los comportamientos deseados al finalizar el proceso formativo y por comprobar (evaluar) que el alumno es capaz de realizar estos comportamientos. De este modo, cualquier plan de estudios o currículo educativo deberá incluir el listado de competencias que debe desarrollar o adquirir, además de describir cómo se evaluará cada una de ellas.

La Fundación Educación Médica ha trabajado en el diseño de planes de estudios de grado y de formación especializada, y también en la definición del perfil profesional competente a partir del cual diseñar planes y estrategias para el desarrollo profesional continuo y procesos de certificación y recertificación profesional. En el transcurso de estos trabajos, siempre hay dos momentos clave: la definición de las competencias [1] y la propuesta de instrumentos de evaluación de estas competencias [2].

En la definición de las competencias se pretende describir los diferentes comportamientos que el profesional debe ser capaz de llevar a cabo. Al tratarse de comportamientos esperados, se suelen utilizar verbos para su definición. Estos verbos deben ser lo menos ambiguos o confusos posible y describir de forma clara e inequívoca el resultado esperado al final de un proceso educativo.

A continuación se recogen las conductas o comportamientos más frecuentes en los planes de estudio de especialistas y de programas de desarrollo profesional continuo en ciencias de la salud. Se han clasificado en función del componente competen- cial más predominante y por orden alfabético para facilitar su utilización. Además, se sugieren los instrumentos de evaluación o comprobación más adecuados en cada caso. En la adecuación de los instrumentos de evaluación de las competencias frecuentemente se identifican dos niveles: uno teórico (el profesional demuestra que sabría qué hacer a partir de viñetas clínicas) y otro basado en la actuación profesional real (el profesional demuestra que es capaz de hacerlo en su contexto real). Siempre que sea posible, se debería optar por la comprobación del comportamiento en contexto real, pero también hay que sopesar las razones de factibilidad en la elección de un instrumento u otro.

\section{Saber (conocimientos)}

\section{Conocer}

El verbo conocer se debe reservar para los contenidos de la especialidad que el profesional debe saber, poder describir o definir conceptos (la historia natural, los principios, los mecanismos de acción, etc.). En medicina está muy extendido el hábito de expresar como 'conocer' lo que en realidad se espera que se sepa hacer. El ejemplo típico es referirse a una técnica (conocer la técnica X) queriendo decir que se debe ser capaz de realizarla correctamente.

El instrumento de evaluación más utilizado para el nivel cognitivo de una competencia son los exámenes escritos, normalmente los tests de respuesta múltiple (TRM) a partir de viñetas clínicas.

\section{Definir}

Véase 'Conocer'.

\section{Describir}

Véase 'Conocer'.

\section{Fundación Educación Médica. E-mail: jpales@ub.edu Conflicto de intereses: No declarado. Competing interests: None declared.} (c) 2015 FEM 


\section{Saber hacer (habilidades)}

\section{Razonamiento clínico}

\section{Analizar}

El profesional distingue y separa las partes de un todo hasta llegar a conocer sus principios o elementos. Otra acepción de este verbo es que el profesional examina unos resultados, una documentación escrita o cualquier realidad susceptible de estudio intelectual. A menudo se relaciona con la literatura científica o con los resultados de pruebas diagnósticas y terapéuticas.

Para comprobar esta capacidad, lo más adecuado es tener un informe escrito por el profesional que refleje el análisis realizado. Este documento se puede incluir en un portafolio.

\section{Derivar}

Este verbo, en el contexto sanitario, se refiere a encaminar o conducir al paciente a otro dispositivo, unidad o profesional. A menudo se usa como sinónimo de remitir.

Esta competencia se puede comprobar en dos niveles diferentes: en teoría, a partir de exámenes escritos con casos clínicos (TRM); o en la práctica del profesional, analizando sus historias clínicas $\mathrm{u}$ otro tipo de registro según la especialidad.

\section{Diagnosticar}

El profesional determina el carácter de una enfermedad o entidad clínica mediante el examen de sus signos y síntomas.

Esta competencia se puede comprobar en dos niveles diferentes: en teoría, a partir de exámenes escritos con casos clínicos (TRM); o en la práctica del profesional, analizando sus historias clínicas $\mathrm{u}$ otro tipo de registro según la especialidad.

\section{Diferenciar}

El profesional distingue, identifica, la diversidad. En los inicios de la formación especializada o cuando se aprenden áreas colindantes a la propia especialidad/tronco, es posible que el profesional sólo deba distinguir entre normal y patológico, o entre urgente y no urgente.

Esta competencia se puede comprobar en dos niveles diferentes: en teoría, a partir de exámenes escritos con casos clínicos (TRM); o en la práctica del profesional, analizando sus historias clínicas $\mathrm{u}$ otro tipo de registro según la especialidad.

\section{Estadificar}

El profesional debe clasificar la extensión y grave- dad de una enfermedad, generalmente una patología tumoral maligna.

Esta competencia se puede comprobar en dos niveles diferentes: en teoría, a partir de exámenes escritos con casos clínicos (TRM); o en la práctica del residente, analizando sus historias clínicas u otro tipo de registro según la especialidad.

\section{Evaluar}

Se refiere a que el profesional es capaz de estimar o apreciar generalmente necesidades del paciente (sociales, nutricionales, de rehabilitación, etc.). A menudo se usa como sinónimo de valorar.

Esta competencia se puede comprobar en dos niveles diferentes: en teoría, a partir de exámenes escritos con casos clínicos (TRM); o en la práctica del profesional, analizando sus historias clínicas u otro tipo de registro según la especialidad.

\section{Identificar}

Esta competencia comporta que el profesional reconoce una patología o entidad clínica. Generalmente se usa como sinónimo de diagnosticar, con el matiz de que no se espera un diagnóstico preciso, porque el profesional está en período de formación o porque son entidades que forman parte de áreas de subespecialización.

Esta competencia se puede comprobar en dos niveles diferentes: en teoría, a partir de exámenes escritos con casos clínicos (TRM); o en la práctica del profesional, analizando sus historias clínicas u otro tipo de registro según la especialidad.

\section{Indicar}

En el ámbito de la medicina, este verbo se asocia a señalar un tratamiento o prueba complementaria de diagnóstico o tratamiento.

Esta competencia se puede comprobar en dos niveles diferentes: en teoría, a partir de exámenes escritos con casos clínicos (TRM); o en la práctica del profesional, analizando sus historias clínicas u otro tipo de registro según la especialidad.

\section{Instaurar}

Poco utilizado, es sinónimo de establecer. Un ejemplo sería 'instaurar medidas preventivas'

Esta competencia se puede comprobar en dos niveles diferentes: en teoría, a partir de exámenes escritos con casos clínicos (TRM); o en la práctica del profesional, analizando sus historias clínicas u otro tipo de registro según la especialidad.

\section{Integrar}

Este comportamiento implica que el profesional cons- 
truye un todo a partir de diferentes partes. Un ejemplo puede ser 'integrar resultados de diferentes pruebas diagnósticas específicas'.

Esta competencia se puede comprobar en dos niveles diferentes: en teoría, a partir de exámenes escritos con casos clínicos (TRM); o en la práctica del profesional, analizando sus historias clínicas $u$ otro tipo de registro según la especialidad. Cabría una tercera posibilidad, con la observación en una situación de supervisión directa en la que el profesional en formación argumentara sus decisiones o conclusiones.

\section{Interpretar}

El profesional explica el significado de algo, generalmente pruebas complementarias o resultados estadísticos.

Esta competencia se puede comprobar en dos niveles diferentes: en teoría, a partir de exámenes escritos con casos clínicos (TRM); o en la práctica del profesional, analizando sus historias clínicas u otro tipo de registro según la especialidad.

\section{Prescribir}

El profesional ordena o indica los productos terapéuticos o remedios para el paciente. Generalmente se debe indicar, además del producto, la dosis, la vía de administración y la periodicidad. Es sinónimo de recetar.

Esta competencia se puede comprobar en dos niveles diferentes: en teoría, a partir de exámenes escritos con casos clínicos (TRM); o en la práctica del profesional, analizando sus historias clínicas u otro tipo de registro según la especialidad.

\section{Prevenir}

El profesional debe ser capaz de anticiparse a una patología, complicación, secuela, etc.

Esta competencia se puede comprobar en dos niveles diferentes: en teoría, a partir de exámenes escritos con casos clínicos (TRM); o en la práctica del profesional, analizando sus historias clínicas u otro tipo de registro según la especialidad.

\section{Recetar}

Véase 'Prescribir'.

\section{Redactar}

El verbo generalmente hace referencia a informes de diversos tipos: de alta, de derivación, etc.

La mejor manera de comprobar que se sabe hacer es la revisión de los informes realizados por los profesionales.

\section{Remitir}

Véase 'Derivar'.

\section{Tratar}

En el ámbito de la medicina, es un verbo altamente utilizado porque corresponde a uno de los fines más importantes de la profesión. Significa aplicar los medios adecuados para curar o aliviar una enfermedad, sus complicaciones y sus secuelas si las hubiera.

Esta competencia se puede comprobar en dos niveles diferentes: en teoría, a partir de exámenes escritos con casos clínicos (TRM); o en la práctica del profesional, analizando sus historias clínicas u otro tipo de registro según la especialidad.

\section{Valorar}

Véase 'Evaluar'.

\section{Comportamientos}

\section{Aconsejar (dar consejo)}

El profesional da su parecer o dictamen sobre una o varias conductas que se deben seguir. Un ejemplo frecuente es 'dar consejo genético'.

La comprobación de esta competencia pasa por preguntar a las personas que reciben este consejo. El instrumento más adecuado es el que recoge la opinión de los distintos actores, la evaluación $360^{\circ}$. En este caso, con preguntar a los destinatarios del consejo sería suficiente. Por su mayor factibilidad, el instrumento más utilizado en la actualidad son rúbricas válidas y fiables usadas por observadores entrenados.

\section{Actuar}

El verbo implica acción. El profesional ejerce funciones propias de su contrato laboral. Generalmente, en este tipo de competencias es importante el cómo se debe actuar. Por ejemplo, actuar según los principios éticos.

La comprobación de esta competencia pasa por preguntar a las personas que observan la actuación del profesional o el residente. El instrumento más adecuado es el que recoge la opinión de los distintos actores, la evaluación $360^{\circ}$. Sin embargo, por su mayor factibilidad, el instrumento más utilizado en la actualidad son rúbricas válidas y fiables usadas por observadores entrenados.

\section{Adaptar}

El profesional debe acomodar o ajustar algo generalmente a cada situación o a cada paciente. Por ejemplo, adaptar un tipo de tratamiento a diferentes etapas evolutivas de un proceso patológico. 
Esta competencia se puede comprobar en dos niveles diferentes: en teoría, a partir de exámenes escritos con casos clínicos (TRM); o en la práctica del profesional o el residente, analizando sus historias clínicas u otro tipo de registro según la especialidad.

\section{Ajustar}

Véase 'Adaptar'.

\section{Colaborar}

El profesional debe ser capaz de trabajar con otra $u$ otras personas en su actividad.

La comprobación de esta competencia pasa por preguntar a las personas con las que tiene que colaborar. El instrumento más adecuado es el que recoge la opinión de los distintos actores, la evaluación $360^{\circ}$. Sin embargo, por su mayor factibilidad, el instrumento más utilizado en la actualidad son rúbricas válidas y fiables usadas por observadores entrenados.

\section{Comprometerse}

Se trata de una conducta que demuestra la existencia de una obligación contraída o una palabra dada. Frecuentemente se refiere a los valores de la profesión.

El instrumento más adecuado para evaluar esta competencia es la evaluación $360^{\circ}$. Es importante poder distinguir entre las declaraciones de intenciones (que se recogerían a través de exámenes escritos) de las conductas reales en contexto real. También es adecuado no centrarse sólo en la autopercepción, ya que una cosa es lo que se piensa que se hace y otra lo que realmente se hace. Por su mayor factibilidad, el instrumento más utilizado en la actualidad son rúbricas válidas y fiables usadas por observadores entrenados.

\section{Comunicar/comunicarse}

Se trata de un comportamiento complejo y que puede variar en función de las teorías desde las que se parta. El profesional hace partícipe a otra $u$ otras personas de información que él o ella tiene. Se puede comunicar de forma oral o escrita.

La evaluación de la capacidad de comunicarse por escrito consiste en analizar los escritos redactados por el profesional. Estos documentos se pueden presentar en el portafolio del profesional.

La evaluación de la comunicación oral es más compleja. Fundamentalmente, si se desea comprobar si ha habido una comunicación eficaz, es decir, que la información se ha comprendido por el receptor del mensaje, la información fundamental debe provenir de los distintos receptores. El instru- mento más adecuado es el que recoge la opinión de los distintos receptores, la evaluación $360^{\circ}$. Sin embargo, por su mayor factibilidad, el instrumento más utilizado en la actualidad son rúbricas válidas y fiables usadas por observadores entrenados.

\section{Contribuir}

Véase 'Participar'.

\section{Cumplir}

El profesional debe ejecutar o llevar a efecto alguna cosa. Un ejemplo puede ser cumplir con los principios y valores de los sistemas de salud.

El instrumento más adecuado para evaluar esta competencia es la evaluación $360^{\circ}$. Es importante poder distinguir entre las declaraciones de intenciones (que se recogerían a través de exámenes escritos) de las conductas reales en contexto real. También es adecuado no centrarse sólo en la autopercepción, ya que una cosa es lo que se piensa que se hace y otra lo que realmente se hace. Por su mayor factibilidad, el instrumento más utilizado en la actualidad son rúbricas válidas y fiables usadas por observadores entrenados.

\section{Diseñar}

Esta acción normalmente se refiere a la elaboración de programas o proyectos. Un ejemplo puede ser diseñar un programa de educación para los pacientes.

La evaluación de esta competencia se basa en aportar documentación que muestre esta actividad, un diseño de programa o proyecto, que se puede recoger en el portafolio del profesional.

\section{Informar}

Véase 'Comunicar'.

\section{Llegar a acuerdos}

Se refiere a adoptar una decisión de común acuerdo entre dos o más partes (con el paciente, con sus familiares o cuidadores, con otros profesionales, etc.).

El instrumento más adecuado para evaluar esta competencia es la evaluación $360^{\circ}$.

\section{Obtener}

La mayoría de las veces hace referencia al consentimiento informado del paciente o sus familiares. El profesional debe conseguir el permiso del paciente o sus familiares para poder realizar algún tipo de procedimiento diagnóstico o terapéutico.

Esta competencia se puede comprobar a partir del análisis de las historias clínicas.

Véase también ‘Obtener' en el apartado de técnicas y procedimientos. 


\section{Participar}

El profesional ayuda, toma parte o concurre con otros al logro de algún fin. Un ejemplo es la investigación que se pueda llevar a cabo en la unidad docente. También se usa la expresión 'contribuir' para expresar esta competencia.

La evaluación de esta competencia se basa en aportar documentación que muestre esta actividad, que se puede recoger en el portafolio del profesional.

\section{Planificar}

El profesional establece los pasos para la ejecución de una acción, por ejemplo, planificar los cuidados.

La evaluación de esta competencia se basa en aportar documentación que muestre este tipo de actividad, que se puede recoger en un portafolio.

\section{Registrar}

El profesional anota o señala datos o acciones llevadas a cabo en algún tipo de documento o aplicativo informático según su especialidad.

Esta competencia se debe comprobar a partir del análisis de la calidad de los registros efectuados por el profesional.

\section{Relacionarse}

Véase 'Comunicar'.

\section{Seleccionar}

El profesional debe escoger entre diferentes opciones. Algunos ejemplos son 'seleccionar la vía de comunicación, la fuente de progenitores, los componentes sanguíneos para el tratamiento de....

Esta competencia se puede comprobar en dos niveles diferentes: en teoría, a partir de exámenes escritos con casos clínicos (TRM); o en la práctica del profesional o el residente por observación directa, que puede ser más o menos estructurada según cada acción que haya que observar. La ejecución se puede observar en contexto simulado o real.

\section{Trabajar}

El profesional es capaz de ejercer una función. Esta acción se refiere con frecuencia al trabajo en equipo.

La evaluación de este comportamiento se basa en recoger la opinión de los otros agentes implicados. El instrumento más adecuado para evaluar esta competencia es la evaluación $360^{\circ}$. Por su mayor factibilidad, el instrumento más utilizado en la actualidad son rúbricas válidas y fiables usadas por observadores entrenados.

\section{Usar/utilizar}

El profesional debe hacer servir una cosa para algo.
Algunos ejemplos son 'utilizar racionalmente los medicamentos, las escalas de medición del dolor, etc.'.

La comprobación de este tipo de competencias dependerá de lo 'usado' en cada caso. En el ejemplo del uso racional de medicamentos, se puede comprobar en dos niveles diferentes: en teoría, a partir de exámenes escritos con casos clínicos (TRM); o en la práctica del profesional, analizando sus historias clínicas u otro tipo de registro según la especialidad. En el ejemplo de las escalas de dolor, se puede comprobar por observación directa, que puede ser más o menos estructurada según cada acción que haya que observar. La ejecución se puede observar en contexto simulado o real. Esto permite valorar cómo el profesional usa estas escalas. Para valorar cuándo (pertinencia del uso) las usa, el análisis de historias clínicas del profesional es un método adecuado.

\section{Técnicas y procedimientos}

\section{Aplicar}

El profesional debe poner en práctica un conocimiento, una medida o un principio, es decir, realizar en un caso particular lo que se ha establecido en general. Se trata de un comportamiento que puede tener diferentes concreciones: aplicar criterios, estrategias, normas, principios, escalas $u$ otros instrumentos de medida.

La mayoría de estas acciones referidas a criterios, principios, etc., se pueden comprobar a partir del análisis de historias clínicas realizadas por el profesional. Por otro lado, cuando se trata de procedimientos y técnicas concretos, se han desarrollado instrumentos concretos de observación directa válidos y fiables (Direct Observation of Procedural Skills).

\section{Colocar}

El profesional debe ser capaz de poner a alguien o algo en su debido lugar. En medicina, un ejemplo puede ser la colocación de un acceso venoso central.

Este tipo de habilidad se comprueba por observación directa en contexto real o en modelos o simuladores. Cuando sea el caso, se pueden utilizar instrumentos concretos de observación directa válidos y fiables (Direct Observation of Procedural Skills).

\section{Hacer}

Esta competencia hace referencia a la ejecución de técnicas o procedimientos concretos. El ejemplo más frecuente es 'hacer una historia clínica completa.' Es sinónimo de realizar, por ejemplo, 'realizar una exploración física.' 
Este tipo de competencia se comprueba por observación directa, que puede ser más o menos estructurada según cada acción que haya que observar. La ejecución se puede observar en contexto simulado o real.

\section{Liberar}

El profesional debe ser capaz de hacer que algo quede libre. Un ejemplo es 'liberar la vía aérea'.

Este tipo de competencia se comprueba por observación directa, que puede ser más o menos estructurada según cada acción que haya que observar. La ejecución se puede observar en contexto simulado o real.

\section{Manipular}

Esta acción se refiere a que el profesional opera con las manos o con cualquier instrumento. Un ejemplo podría ser 'manipular células madre'.

Esta competencia se puede comprobar a través de la observación directa, más o menos estructurada.

\section{Obtener}

Dentro de este apartado de técnicas y procedimientos, la acción de obtener tiene que ver con extraer un material o un producto de cierta manera, por ejemplo, 'obtener muestras'.

Este tipo de competencia se comprueba por observación directa, que puede ser más o menos estructurada según cada acción que haya que observar. La ejecución se puede observar en contexto simulado o real.

Véase también 'Obtener' en el apartado de comportamientos.

\section{Realizar}

Véase 'Hacer'.

\section{Saber ser (actitudes)}

\section{Respetar}

El profesional debe tener en consideración, por ejemplo, los derechos del paciente.

El instrumento más adecuado para evaluar esta competencia es la evaluación $360^{\circ}$. Es importante poder distinguir entre las declaraciones de intenciones (que se recogerían a través de exámenes escritos) de las conductas reales en contexto real. También es adecuado no centrarse sólo en la autopercepción, ya que una cosa es lo que se piensa que se hace y otra lo que realmente se hace. Por su mayor factibilidad, el instrumento más utilizado en la actualidad son rúbricas válidas y fiables usadas por observadores entrenados.

\section{Verbos que se deben evitar por ser redundantes con el proceso formativo}

Los verbos 'adquirir,' 'aprender' y 'desarrollar habilidades o competencias' aparecen a menudo en programas de formación.

Estas expresiones son redundantes con el proceso educativo de aprendizaje. La idea no es describir el proceso que se debe realizar, sino el resultado esperado al finalizar el período de formación. La definición de la competencia debe describir directamente el comportamiento que el profesional debe ser capaz de llevar a cabo.

\section{Bibliografía}

1. Palés J. Objetivos educativos, resultados del aprendizaje y competencias. Currículos basados en competencias. In Millán J, Palés J, Morán J, eds. Principios de educación médica. Madrid: Editorial Médica Panamericana; 2015. p. 88-96.

2. Nolla M. La evaluación en educación médica. Principios básicos. Educ Med 2009; 12: 223-9. 